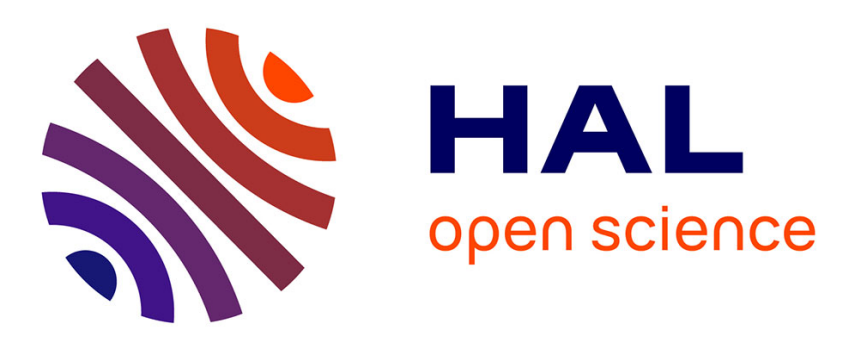

\title{
Automated Detection of Microaneurysms Using Scale-Adapted Blob Analysis and Semi-Supervised Learning
}

Kedir Adal, Désiré Sidibé, Sharib Ali, Edward Chaum, Thomas Karnowski, Fabrice Mériaudeau

\section{To cite this version:}

Kedir Adal, Désiré Sidibé, Sharib Ali, Edward Chaum, Thomas Karnowski, et al.. Automated Detection of Microaneurysms Using Scale-Adapted Blob Analysis and Semi-Supervised Learning. Computer Methods and Programs in Biomedicine, 2014, pp.1-22. 10.1016/j.cmpb.2013.12.009 . hal-00931220

\section{HAL Id: hal-00931220 \\ https://u-bourgogne.hal.science/hal-00931220}

Submitted on 15 Jan 2014

HAL is a multi-disciplinary open access archive for the deposit and dissemination of scientific research documents, whether they are published or not. The documents may come from teaching and research institutions in France or abroad, or from public or private research centers.
L'archive ouverte pluridisciplinaire HAL, est destinée au dépôt et à la diffusion de documents scientifiques de niveau recherche, publiés ou non, émanant des établissements d'enseignement et de recherche français ou étrangers, des laboratoires publics ou privés. 


\title{
Automated Detection of Microaneurysms Using Scale-Adapted Blob Analysis and Semi-Supervised Learning
}

\author{
Kedir M. Adal ${ }^{a, *}$, Désiré Sidibéa, Sharib Alia, Edward Chaum,M.D. b, \\ Thomas P. Karnowski ${ }^{\mathrm{c}}$, Fabrice Mériaudeau ${ }^{\mathrm{a}}$ \\ ${ }^{a}$ Université de Bourgogne, Laboratoire Le2i UMR CNRS 6306, Le Creusot, 71200, France \\ ${ }^{b}$ Hamilton Eye Institute, U. Tennessee Health Sciences Center, Memphis TN, USA \\ ${ }^{c}$ Oak Ridge National Laboratory, Oak Ridge TN, USA
}

\begin{abstract}
Despite several attempts, automated detection of microaneurysm (MA) from digital fundus images still remains to be an open issue. This is due to the subtle nature of MAs against the surrounding tissues. In this paper, the microaneurysm detection problem is modeled as finding interest regions or blobs from an image and an automatic local-scale selection technique is presented. Several scale-adapted region descriptors are then introduced to characterize these blob regions. A semi-supervised based learning approach, which requires few manually annotated learning examples, is also proposed to train a classifier to detect true MAs. The developed system is built using only few manually labeled and a large number of unlabeled retinal color fundus images. The performance of the overall system is evaluated on Retinopathy Online Challenge (ROC) competition database. A competition performance measure (CPM) of 0.364 shows the competitiveness of the proposed system against state-of-the art techniques as well as the applicability of the proposed features to analyze fundus images.
\end{abstract}

Keywords: Microaneurysms, diabetic retinopathy, fundus image, semi-supervised learning, blobs, scale-space.

\footnotetext{
*Corresponding author: Tel.:+33614386360

Email address: KedirAdal@gmail.com (Kedir M. Adal)
} 


\section{Introduction}

Diabetic Retinopathy (DR) which damages the retinal blood vessels is one of the most common causes of blindness in the developed world. Microaneurysms (MAs) are among the early symptoms of DR that can be seen as round dark-red structures in digital color fundus images of retina. Early detection of microaneurysms facilitates timely treatment of DR and limits further complications in the retina. In recent years, automated computeraided detection and diagnosis (CAD) of MAs has attracted many researchers due to its low-cost and versatile nature for public screening applications [1]. However, the variations in size, shape, presence of other retina vascular structures, and illumination variation of fundus images make the design of an accurate CAD system very challenging.

Automatic detection of MAs from fluorescein angiograms was the earliest published work [2] in this research domain. A mathematical morphology based approach using top-hat transform with a linear structural element is used to remove connected vasculatures and other retinal structures. Spencer et al. [3] and Frame1998 et al. [4] have extended this approach by adding shade correction as a pre-processing and match-filters of different sizes to detect MAs. However, the risk associated with intravenous contrast agents limit the usage of fluorescein angiograms for public DR screening programs [5]. On the other hand, recent developments on digital color fundus cameras avoid such a risk and facilitate CAD systems design.

Generally, the current state-of-the-art automated MA detection CAD system design involves three fundamental steps: pre-processing, candidate selection, and MA classification. In fundus imaging, the difficulty in uniformly illuminating the retina results in non-uniform illumination and shading effect [1]. The first step in analyzing such images is thus to compensate for this non-uniform illumination. The green channel of color fundus images is commonly used and analyzed for illumination correction. This is due to its higher contrast between retinal blood vessels and background whereas the remaining red and blue channels suffer from saturation and dark color respectively.

The primarily technique of fundus image contrast normalization employs either global or local histogram equalization [6]. Background estimation and subtraction methods are the most commonly used techniques to correct shading effects [7]. This method uses the green channel of the image to estimate the "background" image using a large median filter of size $25 \times 25$ and sub- 
tract it from the green channel resulting in the shade corrected image. Cree et al. [8] map the shade corrected (background free) image to have a specified mean and variance which is estimated from a reference image. Foracchia et al. [9] further addressed intra and inter-image variability by estimating local variations in contrast and luminosity in the background image and subsequently compensating this variation for the whole image.

Microaneurysm candidate selection is among the most crucial steps towards efficient automated detection methods. A mathematical morphology based candidate microaneurysm extraction method is proposed by Spencer et al. [7] and Frame et al. [4]. The proposed approach removes bright-lesions by setting all positive pixel values of shade corrected image to zero. Morphological top-hat transform is used to suppress large connected dark regions and vessels from further processing. To increase the contrast of the remaining candidate objects, a 2D Gaussian matched-filter is applied. Finally, region growing is performed to increase the size of actual microanueyrsm candidates. Niemeijer et al. [5] exploited the same approach together with a pixel level supervised classification method using multi-scale Gaussian derivative features and KNN classifier. To suppress large connected regions, connectedcomponent analysis is applied and regions with more than 300 pixels are excluded. Finally, the two candidate extraction methods are combined [5]. Walter et al. [10] extracted micraoneurysm candidates using top-hat transform preceded by gray-scale diameter closing operation. Other approaches combine different pre-processing steps to remove retinal structures such as optic disk, fovea, and vasculatures and analyze the remaining regions to detect retinal lesions $[11,12]$.

Sanchez et al. [13] proposed a three class Gaussian mixture-model based on the assumption that each pixel belongs to one of the three classes: background, foreground (vessels, lesions and optic disc), and outlier. Lazar et al. $[14,15]$ proposed a method based on the analysis of intensity profile in color fundus images using different scan lines having orientation angle in the range $\theta \in[-90,90]$. For each orientation angle, the scan lines are translated horizontally and vertically to cover all pixels of the image. Each 1D profile is then binarized using an adaptive thresholding by analyzing its local-maxima and height of each peak. A temporal map is constructed to store the number of times a given pixel has been classified as local-maxima or minima. The temporal map is finally smoothed and hysteresis thresholding is applied. Other approaches include a multi-scale Gaussian correlation filtering together with dynamic thresholding scheme [16] and circular Hough-transforms [17]. 
A Gaussian mixture-model based clustering combined with logistic-regression classification method has been proposed to classify microaneurysms at pixel level [13]. The model is built using features extracted from color, shape, and texture of color fundus images. Mizutani et al. [18] proposed a double-ring filter based microaneurysm classification method based on rules and artificial neural network (ANN). The initial detection starts by applying a double-ring filter on the green channel of the image followed by vessel removal. The remaining pixel regions shape, color, and contrast against its surrounding is extracted to train an ANN. Walter et al. [10] also applied a KNN classifier using intensity, shape, and number of pixel features extracted from each candidate region. An accurate segmentation of retinal blood vessels is thus a crucial step and improves the detection of retinal lesions [19]. Several methods have been proposed for the detection and removal of these structures $[20,21]$.

Quellec et al. [22] proposed a microaneurysm detector based on template matching in wavelet space. The proposed method models MAs using 2D rotation-symmetric generalized Gaussian function template. Giancardo et al. [23] proposed a rule-based MA detector using Radon-transformation which exploits the Gaussian ridge looking like structure of MAs in Radon-space. This approach has also been further improved by using SVM supervised classification method on features extracted from Radon-space [24]. Grisan et al. [25] applied a hierarchical Bayesian classification approach to segment dark and bright lesions from fundus images. Ram et al. [26] modeled false MAs as clutters and a successive-clutter-rejection approach is applied to detect true MAs.

Antal et al. [27] proposed a combination of different pre-processing and candidate extractor methods using optimal voting scheme. The selection of optimal ensembles, pre-processing and candidate extractor pairs are optimized using simulated annealing [28]. The final microaneurysms are then found by fusing the candidates of the pairs which are considered as optimal ensemble. This system is so far among the top performing methods in the Retinopathy Online Challenge database of images [29].

\section{Methodology}

Microaneurysms are round dark-red structures caused by thickening of capillary basement membranes within the retina. Apart from being "round dark-red" structures in digital color fundus images, little is known from CAD 


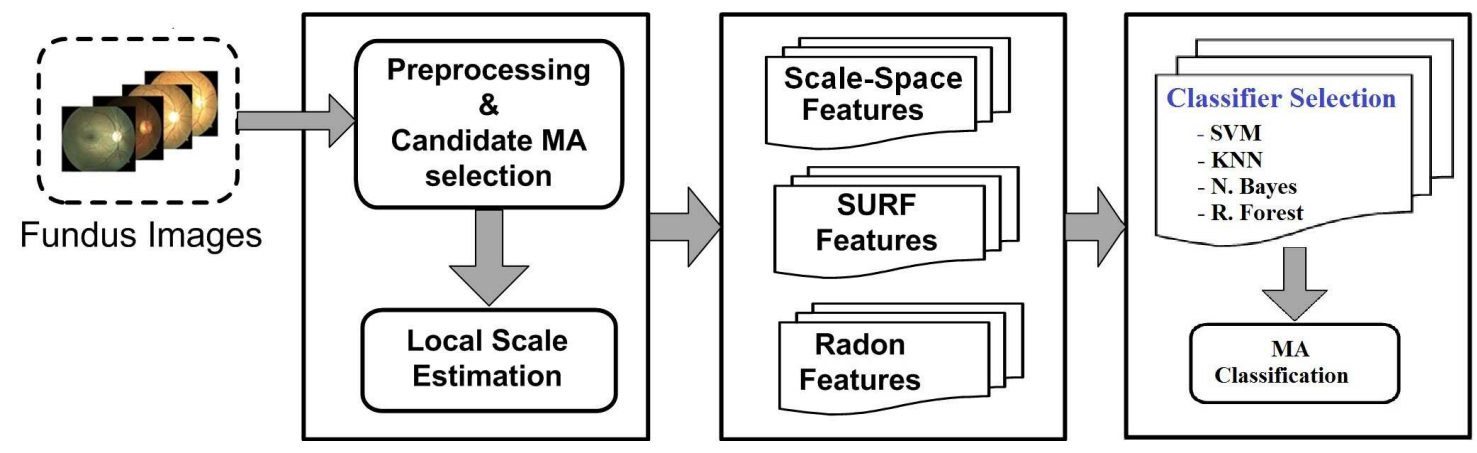

Figure 1: The proposed flow chart of an automated microaneurysm detector CAD system.

designers point of view. The variation in size, shape, intensity, and presence of other retina structures further complicate the problem of detecting MAs from fundus images.

The majority of previous works have focused on dealing with these issues individually. Little effort has been made so far to integrate together these variations in order to have robust MA descriptors; for instance size and appearance of MAs are not well integrated. On the other hand, the task of automated MA detection fits well into one category of interest point or blob detection task. This intuition motivated us to model the MA detection task as a circular blob detection problem from fundus images.

In this paper, we present a robust MA candidate selection technique which is built upon scale-invariant interest-point or blob detection theory. Then several robust scale-adapted microaneurysm feature extraction techniques are discussed in order to capture local image descriptors from fundus images. Moreover, the difficulty of getting manually annotated example images from which learning algorithms can be trained is also discussed. Towards this end, we investigate a semi-supervised machine learning approach which is built up on the notion of exploiting "unlabeled" data together with few "labeled" images to train the final MA classifier system.

Figure 1 shows the overall flow chart of the proposed CAD design for automated MA detection. Each of the processing stages are discussed in the subsequent sections. 


\section{Pre-processing and Candidate Extraction}

The pre-processing and MA candidate extraction stage is the most crucial part in automated detection of diabetic retinopathy. A proper candidate selection algorithm reduces false detection rate as well as computation time; thus it increases accuracy and speeds up the automated detection process.

As a pre-processing step, we applied a singular value decomposition (SVD) based contrast enhancement technique which can reduce the shading effect while increasing the contrast of fundus images [30]. This contrast enhancement method is based on the observation that the singular value distribution of good quality retinal images is equivalent; whereas the corresponding distribution of low quality fundus images is low compared to the good ones as shown in figure 2. The proposed contrast enhancement method is thus done by equalizing all the singular values of any low quality image by the corresponding values of the reference image. An example contrast enhancement result is also shown in figure 3 .

In fundus images, the intensity values at locations corresponding to MAs are locally different from their surrounding regions i.e low in their pixel value. To detect these regions, a local-minima detection method is proposed in our previous work, Adal et al. [30]. The proposed approach exploits the determinant of the Hessian matrix of each pixel on the green channel of the image to extract dark-circular regions. These regions have strong derivatives along both orthogonal directions resulting in higher determinant value as shown in Figure 4.

The Hessian matrix of each pixel $\mathbf{x}=(x, y)$ is:

$$
H(\mathbf{x} ; \sigma)=\left[\begin{array}{ll}
I_{x x}(\mathbf{x} ; \sigma) & I_{x y}(\mathbf{x} ; \sigma) \\
I_{x y}(\mathbf{x} ; \sigma) & I_{y y}(\mathbf{x} ; \sigma)
\end{array}\right],
$$

where each element, $I . .(\mathbf{x} ; \sigma)$, of the matrix is the convolution of the image pixel at location $\mathbf{x}=(x, y)$ along the specified subscript directions with a second-order Gaussian derivative kernel at a scale of $\sigma$. For instance $I_{x x}(\mathbf{x} ; \sigma)$ is defined as:

$$
I_{x x}(\mathbf{x} ; \sigma)=I(\mathbf{x}) * G_{x x}(\sigma),
$$

where $G_{x x}(\sigma)$ is a second-order Gaussian derivative along x-direction.

In general, the response of the Hessian-operator depends on the scale, $\sigma$, at which $I . .(\mathbf{x} ; \sigma)$ is computed. For candidate MA selection, we have 


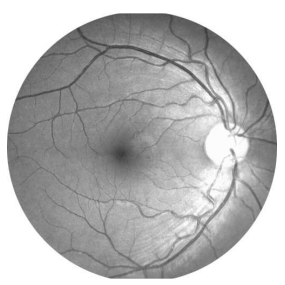

(a) Ref image-

1.

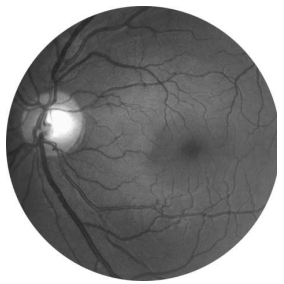

(b) Ref image2.

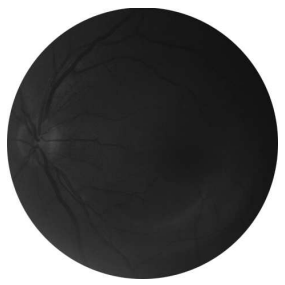

(c) Low c. image.

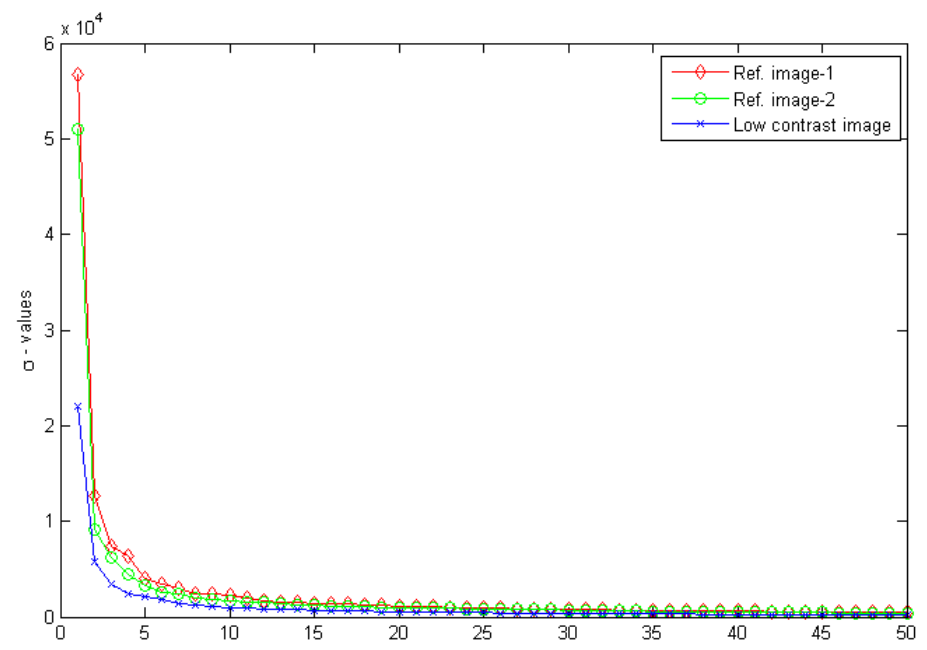

(d) Singular values of images (a)-(c).

Figure 2: Singular value distribution of different contrast fundus images.

applied thresholding on the determinant of Hessian operator at $\sigma=2$, as the majority of MA sizes give a good response at this scale. However, this scale dependency will be further exploited in the next sections to estimate the local-scale at which candidate MAs give can be best characterized. The candidate selection algorithm is summarized in table 1 and an example of selected candidate microaneurysms are shown in figure 5.

\section{Local-Scale Estimation}

Clinically, the size of microaneurysms range from $20-100$ microns and they appear as dark red structures in color fundus images. Moreover, the variation in the resolution of fundus cameras prohibit from using a fixed- 


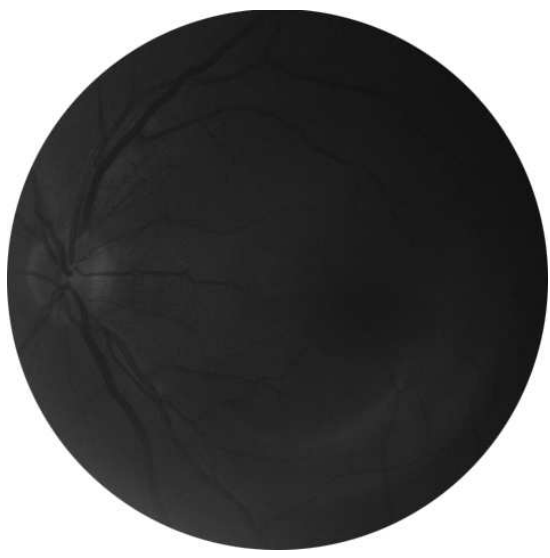

(a)

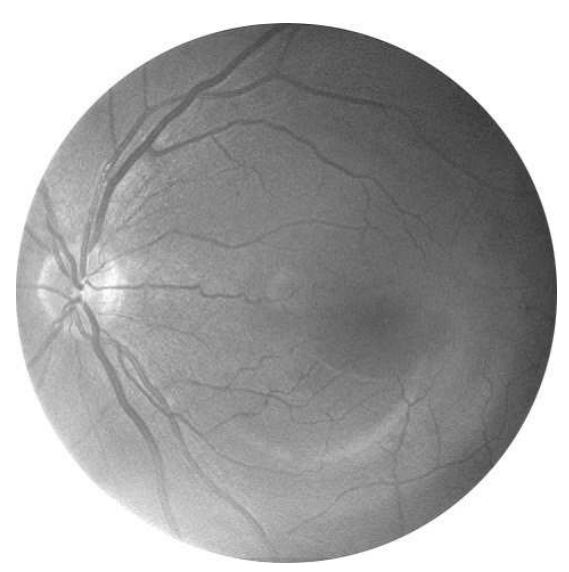

(b)

Figure 3: Example of contrast enhanced image: (a)Input image. (b)Enhanced output image.

scale image analysis approach to detect them. Therefore, handling this scale variation is required.

In computer vision and image processing, a multi-scale representation of image data has been found to be crucial in dealing with a scene having complex structures [31]. This allows to separate different structures within the scene and analyze them at appropriate scales. It is also a well known fact that computing image descriptors at inappropriate scales can result in significantly unstable results. Therefore, estimating local-scale information of candidate MAs plays an important role in order to extract robust features from fundus images. In this work, we adapt the automatic scale selection approach proposed by Lidenberg [31] to fit in our model.

The local-scale of each candidate MA is estimated by computing localmaxima of scale-normalized Hessian response across different scales as shown in Eq. 3 and 4. Note that the optimization is done only with respect to the scale parameter $t$ to find the local maxima as we already have the MA candidate locations $(x, y)$.

$$
\begin{gathered}
(x, y ; \hat{t})=\arg \max _{t}\left(\operatorname{det} H_{\text {norm }}(x, y ; t)\right) \\
\operatorname{det}\left(H_{\text {norm }}(x, y ; t)\right)=t^{2}\left(I_{x x}(t) I_{y y}(t)-I_{x y}(t) I_{y x}(t)\right),
\end{gathered}
$$

where $t=\sigma^{2} \in\left[1.6^{2}, 8^{2}\right]$, and $I_{x x}, I_{y y}$, and $I_{x y}$ are the second-order Gaus- 

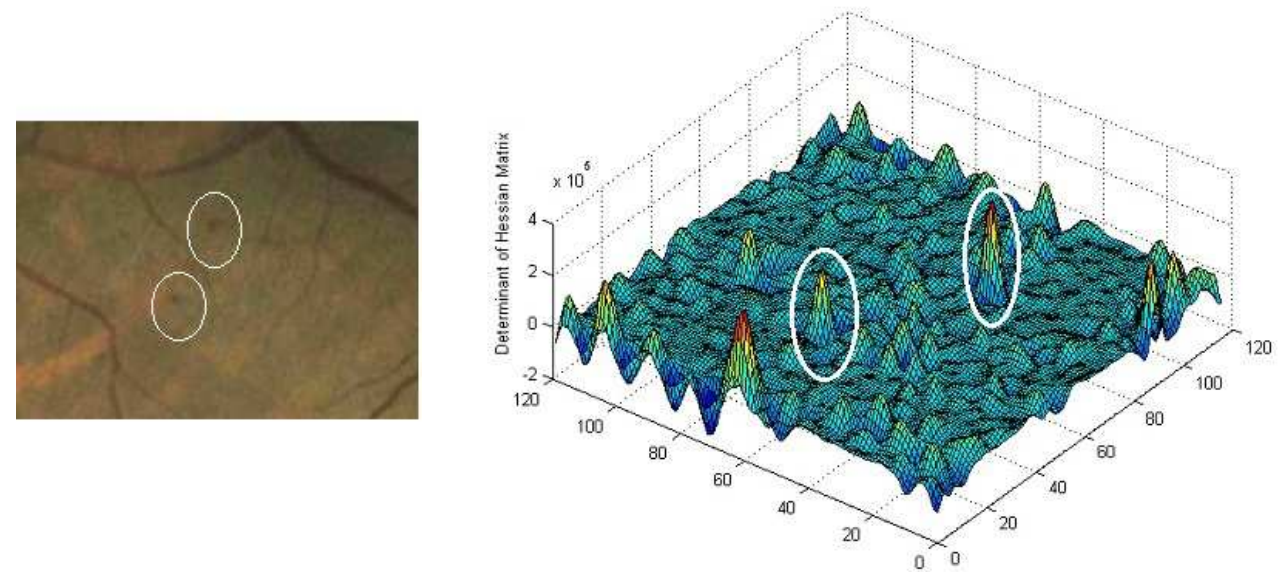

(b) Determinant of Hessian matrix at scale, $\sigma=$

(a) Sample image patch.

2 .

Figure 4: Response of Hessian operator. Microaneurysms and their responses are indicated within the white ellipses.

sian derivatives of the image as defined in Eq. 2. The value of $\hat{t}$ which gives local maximum response is used as an estimated local-scale of the candidate MA at location $(x, y)$.

Figure 6 illustrates this local-scale estimation technique on a sample fundus image in which two MAs are manually annotated by an ophthalmologist. The scale response on figure $6 \mathrm{~b}$ shows that the appropriate scales to extract features of MA-1 and MA-2 are at $\sigma=2$ and $\sigma=1.6$, respectively.

\section{Feature Extraction}

Extracting robust features or candidate region descriptors is an important step for the success of the final classification stage. In this regard, since we modeled candidate MA regions as blob regions within a fundus image, several categories of low-level robust blob descriptors are employed. The next subsections present different proposed features which are found to be good to characterize and classify MAs from fundus images.

\subsection{Scale-Space Features}

The key idea in the scale-space approach is to analyze objects within the image at different scales by representing the image as a one-parameter family 
Algorithm-1: Hessian based microaneurysm Candidate Selection

Given an image $I$ :

- Apply Pre-processing methods.

- Compute the Hessian Matrix, $H$ at $\sigma=2$

- Compute $\lambda_{1}$ and $\lambda_{2}$ of $H$, their absolute ratio, $A r$, and $|H|$ :

$$
\begin{aligned}
& A r=\frac{\left|\lambda_{1}\right|}{\left|\lambda_{2}\right|} \\
& |H|=\lambda_{1} \cdot \lambda_{2}
\end{aligned}
$$

Create candidate mask image $I_{\text {cand }}$ :

$$
I_{\text {cand }}=|H|>\text { Thsd }_{1} \cap A r<T h s d_{2} \cap \lambda_{1}>0 \cap \lambda_{2}>0
$$

Two empirically chosen threshold values $T h s d_{1}=3 \times 10^{4}$ and $T h s d_{2}=2$ have been used.

Table 1: Microaneurysm candidate selection.

of smoothed images [32]. Gaussian function is the most common smoothing function employed in practice. Depending on the differential expression used, the scale-space approach can be used to extract different attributes of objects. As we have pointed out in the previous section, the Hessian-operator, which is crucial in blob detection task, is used as a differential expression to compute feature attributes.

For each candidate regions, the following features are extracted:

- $\operatorname{mean}$ of $\lambda_{1}, \lambda_{2}$, and $|H|$

- $\boldsymbol{m a x}$ of $\lambda_{1}, \lambda_{2}$, and $|H|$

$|H|$ is the determinant of Hessian matrix $H$ and $\left\{\lambda_{1}, \lambda_{2}\right\}$ are the eigenvalues of $H$. A total of 18 features ( 6 for each scale, $\sigma=1,2,4$ ) are extracted.

\subsection{Speeded Up Robust Features (SURF)}

The majority of features come from SURF, which is a well-known technique to extract low-level image descriptors [33]. The two main operation in SURF algorithm are interest point detection and descriptor extraction. Since SURF implicitly uses the Hessian operator to detect interest points, 


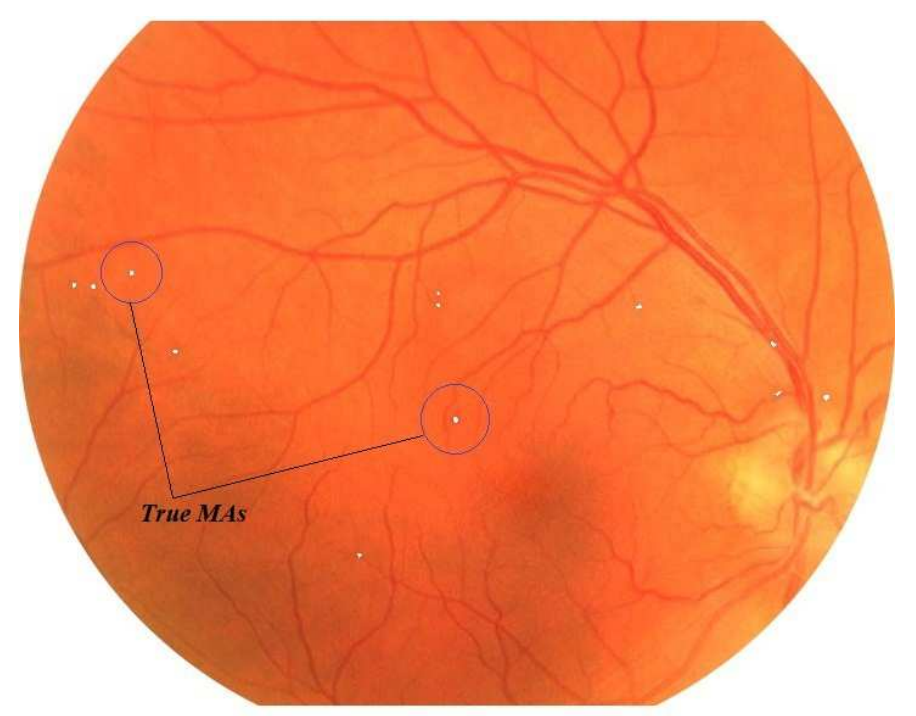

Figure 5: Selected MA candidates (White pixels) using Algorithm-1.

which might not necessarily correspond either to dark or to circular blobs regions, we bypassed this operation. Instead we extract directly the descriptors for the candidate regions which are selected in the pre-processing stage described in section 3.

SURF descriptors are computed by placing a square window of size $20 \sigma$ centered on each candidate MA and dividing it into $4 \times 4$ sub-windows. A Haar-like filter of size $2 \sigma$ are used within each sub-window to compute the filter responses $d x$ and $d y$ as shown in Figure 7 . The estimated local-scale, $\sigma=\sqrt{\hat{t}}$, of each candidate MA (see section 4) is used to define the size of this region for feature computation. Moreover, the orientation or dominant direction of each candidate region is assumed to be along the principal image axes.

Let $x$ and $y$ be the orthogonal directions oriented along the dominant direction (in our case it is assumed to be parallel to the image axes), four Haar-like response descriptors are extracted from each sub-window which are given as follows:

$$
\text { Descriptors }_{\text {sub-win }}=\{\Sigma d x, \Sigma d y, \Sigma|d x|, \Sigma|d y|\}
$$

where $\Sigma \cdot$ and $\Sigma|\cdot|$ refers the summation of the derivatives and absolute value of the derivatives respectively. Therefore, for each candidate MA, a 


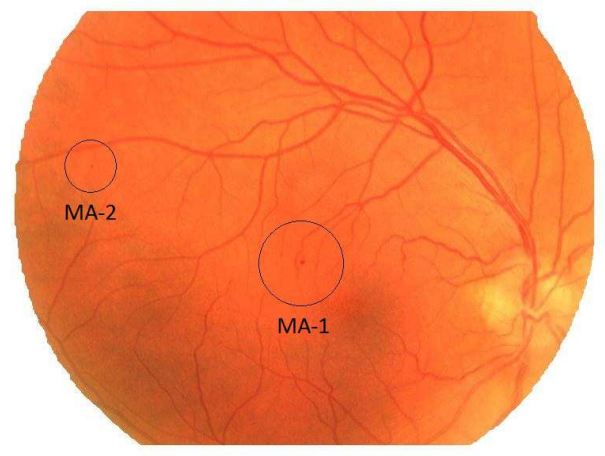

(a) Two manually annotated MAs.

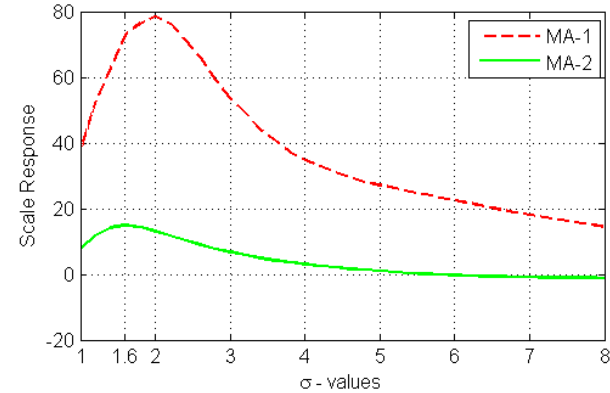

(b) Scale-space response.

Figure 6: Local-scale estimation of two different size MAs (shown in black circles in (a)) and their estimated scale using scale-space approach shown in (b).

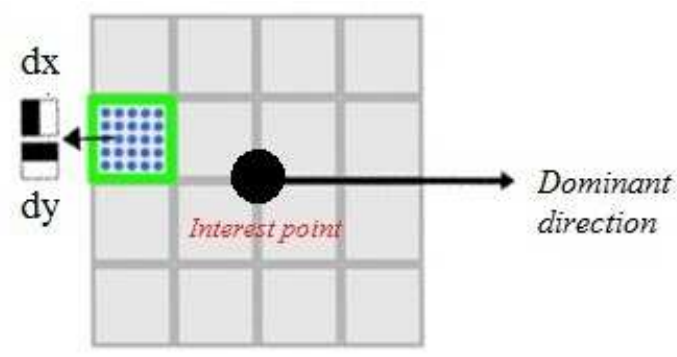

Figure 7: SURF computation window.

total of $64-S U R F$ descriptors $(4 \times 16$ sub-windows $)$ which characterize the region of interest are extracted.

\subsection{Radon-Transform Features}

The Radon transform of an image at pixel location $\mathbf{x}=(x, y)$ can be defined as a series of line integrals from multiple sources along parallel paths in a specific orientation angle $\theta$. Giancardo et al. [23] [24] proposed a method that automatically detects MAs from color fundus images by using Radon transformation. We extend the same approach by applying the transformation on the Intensity Normalized image patch instead of the original image patch. The normalization is done by making the patch to have zero mean and unit variance.

The normalization is required to make the Radon transform illumination invariant. Feasibility study is done on 20 sample image patches where half 


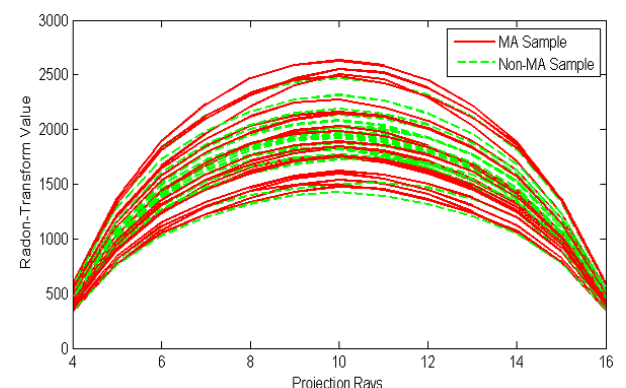

(a) $R_{\mu}(x)$-features of original image.

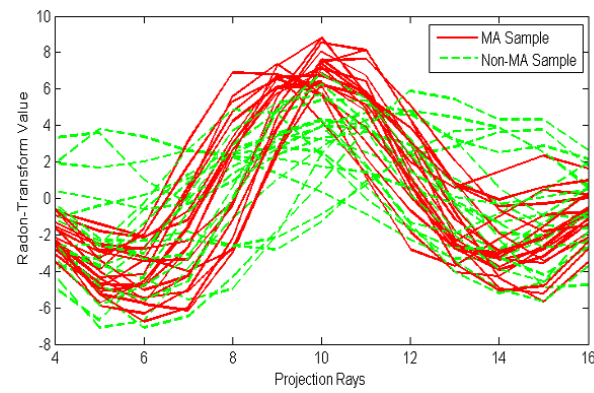

(b) $\quad R_{\mu}(x)$-features of normalized image.

Figure 8: Original vs. Normalized Radon-transform features. The red lines correspond to samples from true-microaneurysm regions and green lines correspond to samples from non-microaneurysm retinal structures.

of them contain one microaneurysm and the remaining half are other retinal structures. The results show that normalizing the intensity gives better discriminant features compared to the original image. Figure 8 shows this observation for the samples selected in the feasibility study.

The Radon transform is then computed from the inverted green channel using a $15 \times 15$ image patch centered at each candidate MA and a projection angle of $\theta \in[0180)$ with a resolution of $\theta_{\text {res }}=5^{\circ}$. Radon metrics that characterize circular-round structures are then computed as follows:

- Mean, $R_{\mu}(x)$, of each projection ray, $x$, at different projection angles:

$$
R_{\mu}(x)=\frac{1}{N} \sum_{n=1}^{N} R\left(n \cdot \theta_{\text {res }}, x\right), 1 \leq x \leq \rho,
$$

where $\rho$ is the number of projection rays and $N=\frac{180}{\theta_{r e s}}$ is the total number of projection angles. $R(\theta, x)$ refers to the Radon transform of ray $x$ at orientation angle $\theta$.

- Standard deviation, $R_{\sigma}(x)$ :

$$
R_{\sigma}(x)=\sqrt{\frac{1}{N} \sum_{n=1}^{N}\left[R\left(n \theta_{\text {res }}, x\right)-R_{\mu}(x)\right]^{2}}
$$


A total of 3 features have been extracted from Radon space. These features are: $\boldsymbol{m a x}\left\{R_{\mu}(x)\right\}, \sigma_{R_{\mu}(x)}, \boldsymbol{m a x}\left\{R_{\sigma}(x)\right\}$. In addition to the features discussed above, the following two additional features are computed directly from each image patch centered at location of interest:

- $\sigma_{I_{\text {patch }}}$ : the standard deviation of the image-patch (computed from the green channel).

- $n P$ :the number of pixels which are similar to the center pixel at the location of interest. This is found by applying region growing by using their intensity as a similarity criterion.

In summary, a total of 87 features (18 Scale-Space + 64 SURF + 3 Radonspace +2 image) have been extracted for each candidate MA.

\section{Microaneurysm (MA) Classification}

Microaneurysm classification is the final stage where true lesions are detected from all candidate regions. This is done using a semi-supervised learning approach which can be built from few supervised information (manually labeled training data) together with a large number of unsupervised information (unlabeled data) [34] [35]. This machine learning approach is especially important in the design of medical CAD systems where getting manually annotated labeled images is very difficult.

Towards this end, we have investigated a self-training and co-training semi-supervised learning approach. In the former approach, a supervised classifier which is trained using few available labeled images re-train itself by including all confidently predicted unlabeled data as a "labeled" data in a recursive manner [35]. Whereas in the later approach, multiple classifiers train each other by looking at different views or features of the samples and forming different classification hypothesis [34].

\section{Experimental Results}

The performance of the overall system is evaluated on both the Retinopathy Online Challenge (ROC) and the University of Tennessee Health Science Center (UTHSC) private database of fundus images. As a training dataset, few manually annotated images consisting of $80 \mathrm{MA}$ samples and 300 unlabeled images from UTHSC database of fundus images have been used. 
Table 2: Optimal classifier-feature pairs.

\begin{tabular}{|l|c|c|}
\hline \hline Classifier & Best Features & Parameters \\
\hline \hline KNN, R. Forest & SURF & K=15, Eucl. Dist. \\
\hline Naïve Bayes & Scale-space & Preceded by PCA \\
\hline SVM & Radon, SURF & Radial Basis Kernel \\
\hline
\end{tabular}

Whereas both training and testing images of ROC-challenge dataset are used for testing purpose.

Four supervised classifiers: K-Nearest Neighbor (KNN), Naïve Bayes, Random Forest, and Support Vector Machines (SVM) [36] are selected as the underlying classifiers. Unlike Antal et al. [28] where an optimization is used to select an optimal pre-processing-candidate selection method, we are only interested in choosing features which are suitable for a given classifer. In this regard, we have conducted an exhaustive performance evaluation on the training dataset to get an optimum classifer-feature pairing summarized in table 2. The selection of a subset of the features for the KNN and Naïve Bayes classifiers is also partially justified as follows:

- Nä̈ve Bayes $\leftrightarrow$ Scale-space: when applying Hessian operator, the response of microaneurysms have been found to have a Gaussian shape, see Figure 4b. Hence, the Naïve Bayes classifier combined with the Gaussian distribution model benefits from this individual feature probability distribution.

- $\boldsymbol{S U R \boldsymbol { F }} \leftrightarrow \boldsymbol{K} \boldsymbol{N} \boldsymbol{N}$ : in point-matching problems, it has been proved that using simple similarity measure, such as euclidean distance, between SURF descriptors of candidate matching points gives good matching criterion [33]. In the same way, using SURF features together with KNN classifier, which works based on the same principle of similarity measure, is an appropriate choice in classifying candidate regions based on their similarity with a true microaneurysm feature.

For each optimal classifier-feature pair summarized in Table 2, a selftraining semi-supervised learning model is applied. The performance of each system is then evaluated on Retinopathy Online Challenge (ROC) training images database. Figure 9 shows the free-response receiver operating characteristic (FROC) curve plot where the system is evaluated on lesion-base. The 


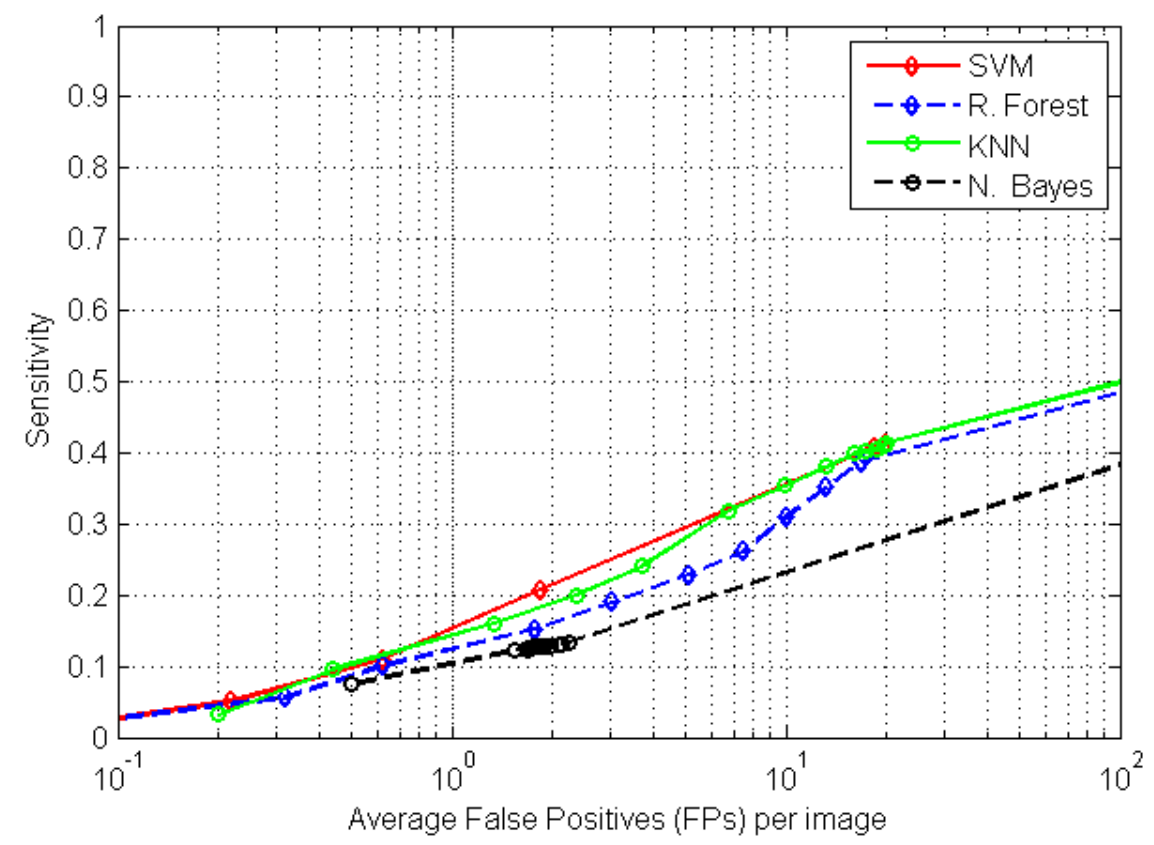

Figure 9: FROC curve using the three classifiers in Self-training mode.

performance evaluation results show that Radon and SURF features coupled with SVM and KNN classifiers outperform the other classifier-feature pairing. Note that the $\mathrm{x}$-axis is plotted in logarithmic scale.

A second set of experiments have also been conducted using co-training approach. The two best performing classifiers i.e SVM and KNN have been chosen to train each other based on their degree of confidence; we discarded the Naïve Bayes and Random Forest classifier due to their low performance results. The performance of each classifier have been improved further especially at medium false positive as shown in figure 10 .

The evaluation results on the ROC training dataset also shows that the proposed MA candidate selection algorithm outperforms previously published methods [27] as shown in table 3. Our method reduces the false candidate detection by about $52 \%$ compared to the best performing technique proposed by Lazar et al. $[14,15]$ with a comparable sensitivity of $44.64 \%$.

We have also participated in the online retinopathy challenge (ROC challenge) by submitting our detection results. The challenge organizers eval- 


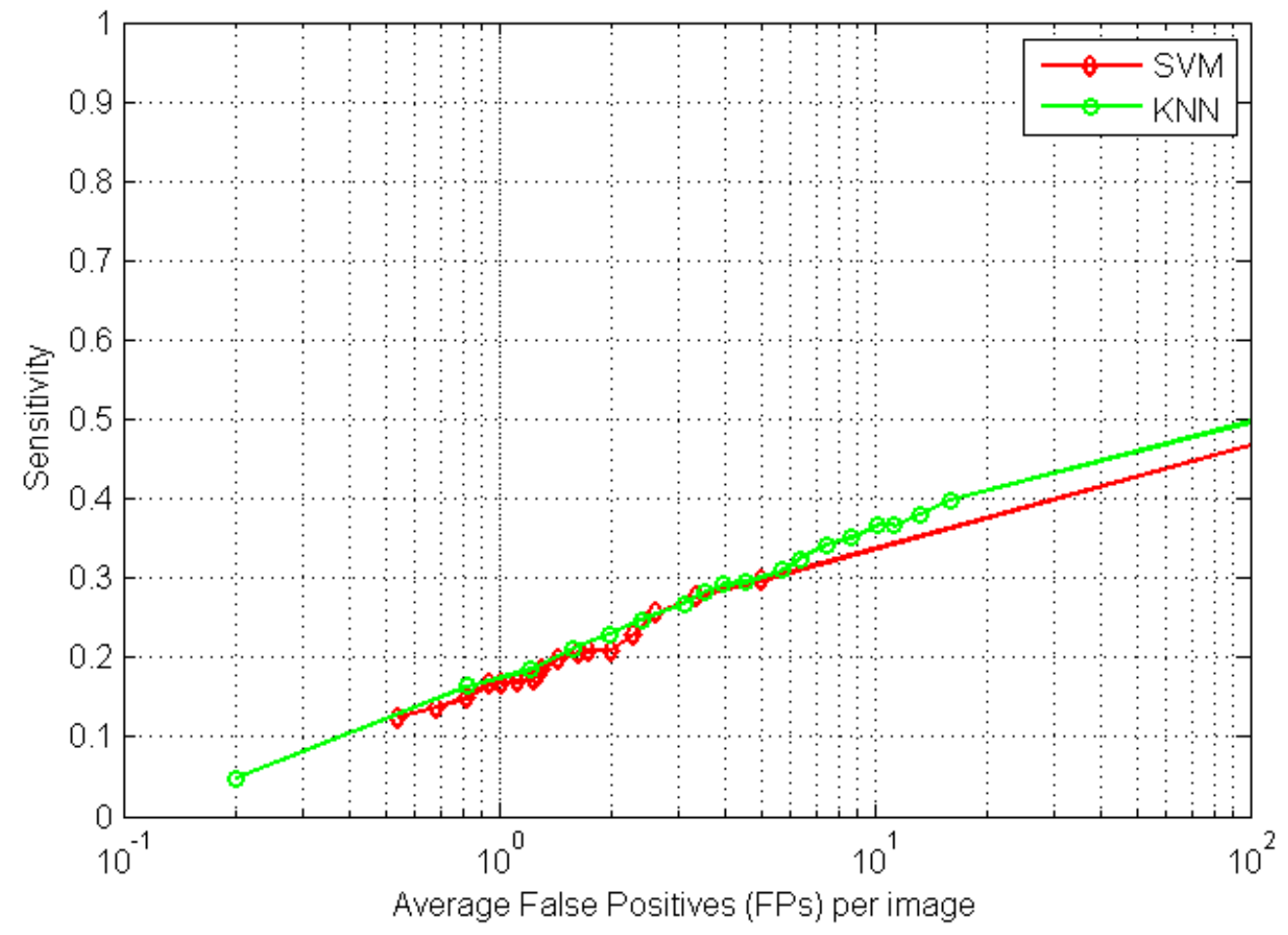

Figure 10: FROC curve of SVM-KNN co-trained classifiers.

uate each participants CAD system and assign a competition performance score (CPM) based on the average sensitivity values at different false positive (FPs) ratios or operating points (OP). Though the organizers provide the full range of FROC values for each participants, the CPM score is assigned only by averaging the sensitivity at operating points $O P_{1}=\left\{\frac{1}{8}, \frac{1}{4}, \frac{1}{2}, 1,2,4,8\right\}$. Table 4 and 5 shows the FROC performance of our system when evaluated on retinopathy challenge testing dataset. Table 6 presents a comparison of the results submitted as of 2009 at two different operating points $O P_{1}$ and $O P_{2}$. Operating point $O P_{2}=\{2,4,8,12,16,20\}$ is included here to show the performance of each system at low and medium range of FPs respectively.

The challenge competition score results show that, our microaneurysm detector can achieve up to $56 \%$ sensitivity at an average false positive rate of 10. This performance is achieved with few manually annotated training samples. Moreover, the increased in performance obtained by using scale-adapted (variable $\sigma$ ) features against fixed scale approach asserts the importance of 
Table 3: Comparison of different candidate extractors. Note that, except our method all the performance values are reported from [27] implementation of each method.

\begin{tabular}{|l|l|l|l|}
\hline \hline Authors & Methodology & Sensiti. & FPs/Img \\
\hline \hline Spencer et al. [7] & Top-hat transform & $12 \%$ & 20.3 \\
\hline Abdelazeem [17] & Circular Hough-transform & $28 \%$ & 505.85 \\
\hline Walter et al. [10] & Diameter closing & $36 \%$ & 154.42 \\
\hline Zhang et al. [16] & Multiple-Gaussian mask & $33 \%$ & 328.3 \\
\hline Lazar et al. [14, 15] & Cross-section profile & $48 \%$ & 73.94 \\
\hline Our method & Hessian Operator & $\mathbf{4 4 . 6 4 \%}$ & $\mathbf{3 5 . 2 0}$ \\
\hline
\end{tabular}

Table 4: FROC results on of retinopathy challenge testing dataset at low false positives per image.

\begin{tabular}{|l|c|c|c|c|c|c|c|}
\hline \hline FPs/Img. & $\frac{1}{8}$ & $\frac{1}{4}$ & $\frac{1}{2}$ & 1 & 2 & 4 & 8 \\
\hline Sensiti. & 0.204 & 0.255 & 0.297 & 0.364 & 0.417 & 0.478 & 0.532 \\
\hline
\end{tabular}

scale estimation to extract robust descriptors from fundus images. The score obtained at $\mathrm{OP}_{2}$ also shows the competitive performance of the proposed approach (CPM of 0.538) against state of the art MA detection systems at medium FPs while being trained with only few training images.

An evaluation is also performed on a publicly available dataset DIARETDB1 [38] which consisits of 89 fundus images manually annotated by four different experts. Since there is a disagreement between the four experts annotations, a consensus of $75 \%$ agreement is considered to label a region as MA and results in a total of 182 MAs. Among the 89 fundus images, 28 images are given for training and the remaining for testing purpose. In our evaluation, we have used all of them for testing purpose. The FROC results shows that our method can achieve a sensitivity of $64.62 \%$ at an average false positive rate of 10 while being trained on a different dataset. This Figure 11 shows the overall result for DIARETDB1 dataset.

The performance of the proposed CAD system has also been evaluated on a private retina image database which is collected from a teleophtamology network managed by the Department of Ophthalmology at the University of Tennessee Health Science Center (UTHSC) in Memphis, Tennessee. This evaluation is done to assess the feasibility of the system to be used in a standalone mode in public DR-screening systems where patients can quickly be screened for the disease in their primary care doctor's office and other 
Table 5: FROC results on of retinopathy challenge testing dataset at medium range of false positives per image.

\begin{tabular}{|l|c|c|c|c|c|c|}
\hline \hline FPs/Img. & 2 & 4 & 8 & 12 & 16 & 20 \\
\hline Sensiti. & 0.417 & 0.478 & 0.532 & 0.586 & 0.606 & 0.606 \\
\hline
\end{tabular}

Table 6: Competition performance measure(CPM) of Retinopathy online challenge [37] at different operating points, $\mathbf{O P}_{\mathbf{1}}$ and $\mathbf{O P}_{\mathbf{2}}$.

\begin{tabular}{|l|c|c|}
\hline Group Name & CPM at $\mathbf{O P}_{\mathbf{1}}$ & $\mathbf{C P M}$ at $\mathbf{O P}_{\mathbf{2}}$ \\
\hline \hline Waikato & 0.206 & 0.335 \\
\hline IRIA-Group & 0.264 & 0.503 \\
\hline Fujita Lab & 0.310 & 0.468 \\
\hline GIB Valladolid & 0.322 & 0.514 \\
\hline OKmedical II & 0.369 & 0.502 \\
\hline ISMV & 0.375 & 0.469 \\
\hline LaTIM & 0.381 & 0.565 \\
\hline Niemeijer & 0.395 & 0.558 \\
\hline DRSCREEN & 0.434 & 0.666 \\
\hline Our Method $(\sigma=1.6)$ & $\mathbf{0 . 3 3 8}$ & $\mathbf{0 . 5 2 3}$ \\
\hline Our Method $(\sigma-$ variable $)$ & $\mathbf{0 . 3 6 4}$ & $\mathbf{0 . 5 3 8}$ \\
\hline
\end{tabular}

remote sites. This is very crucial in reducing the amount of time spent by ophtamologists to screen out each and every retinal image.

A set of 50 images where 37 of them are diagnosed with diabetic retinopathy and the remaining 13 images are free of diabetic retinopathy have been used as an evaluation set. Except the category of each image, a lesion-based annotation is not given for all the images. The performance of the CAD system is then evaluated on its ability to categorize these images either as "Normal" or "Abnormal". Figure 12 shows image level detection result using the two best classifier models.

The result shows that when the SVM classifier model is used, the system can achieve a maximum sensitivity of $81.08 \%$ at a specificity of $92.31 \%$. Moreover, the area under curve (AUC) of the KNN classifier can reach up to 0.8805 . The same evaluation has also be done for the 89 images of DIARETDB1 dataset and an AUC of 0.7228 is achieved using KNN classifier as it is shown in figure 13. This performance is specially appreciated if the system is to be operated under the supervision of clinicians. In general, the overall performance of the proposed CAD system is highly promising to be used in public diabetic retionpathy screening programs and teleophtamology 


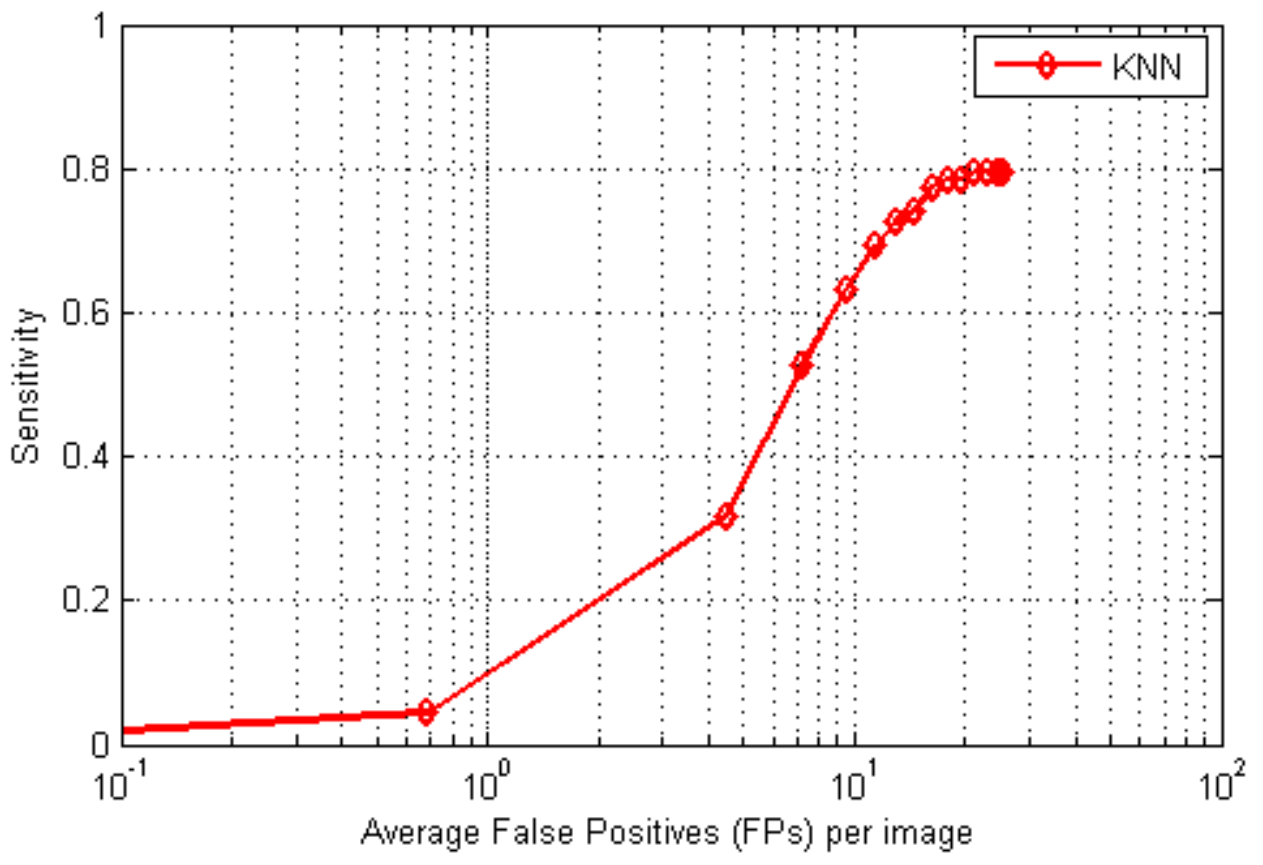

Figure 11: FROC curve of DIARETDB1 dataset.

networks; where computers are being used to do automated diagnosis and generate medical reports for clinicians review.

\section{Conclusion}

In this paper, an automated early diabetic retinopathy screening tool which can be used in public DR screening programs via microaneurysm detection is proposed. We have presented an approach where the microaneurysm detection problem is modeled as finding interest points or blobs from an image. An automatic local-scale estimation technique and several blob descriptors are then introduced to extract scale-adapted features from these blob regions. Moreover, the applicability of semi-supervised learning approach for MA classification is investigated. This approach is especially important in designing medical CAD systems where getting manually annotated examples is among the main design challenge. Only few manually labeled and a large number of unlabelled fundus images are used to train the final MA detection classifier. 


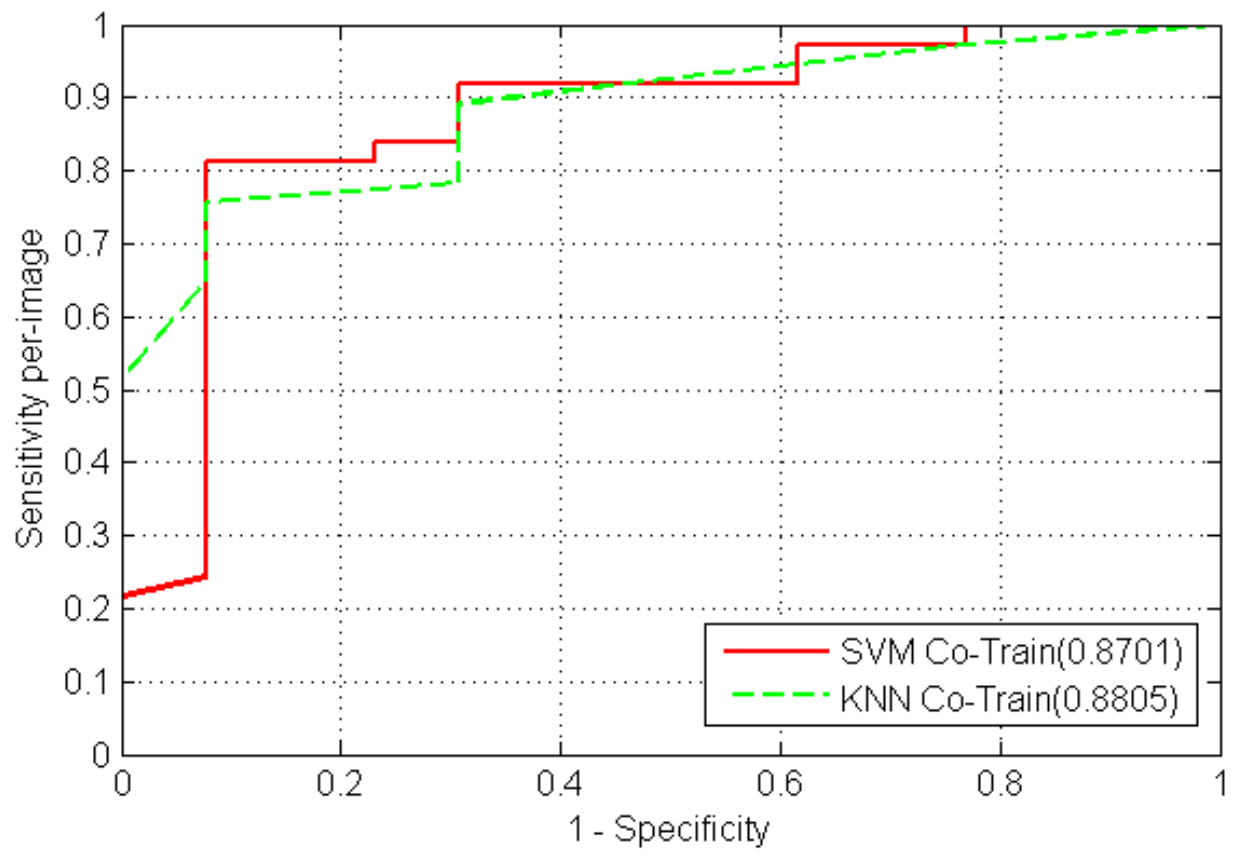

Figure 12: Performance Evaluation on UTHSC Database.

The performance of the overall system is evaluated on the ROC-Challenge and UTHSC private databases. A competition performance measure (CPM) of 0.364 shows the competitiveness of the proposed system against state-ofthe art techniques while using a very few number of manually annotated images for training. Moreover, the proposed approach shows the promising applicability of scale-adapted blob descriptors in analyzing fundus images where illumination variation and noise are prevalent challenges. The fast computation time of these descriptors also facilitates to speed-up the overall fundus image analysis.

As a final closing remark, we would like to point out that we have noticed a significant difference in performance of different state of the art MA detectors when evaluated at different fundus image databases. This is mainly due to several factor and variation among fundus image datasets such as image contrast, resolution, and number of training and testing images included. In our proposed system, we have shown that being trained on few images of independent dataset, our system can achieve a comparable performance when tested on other datasets. This is especially crucial for the proposed 


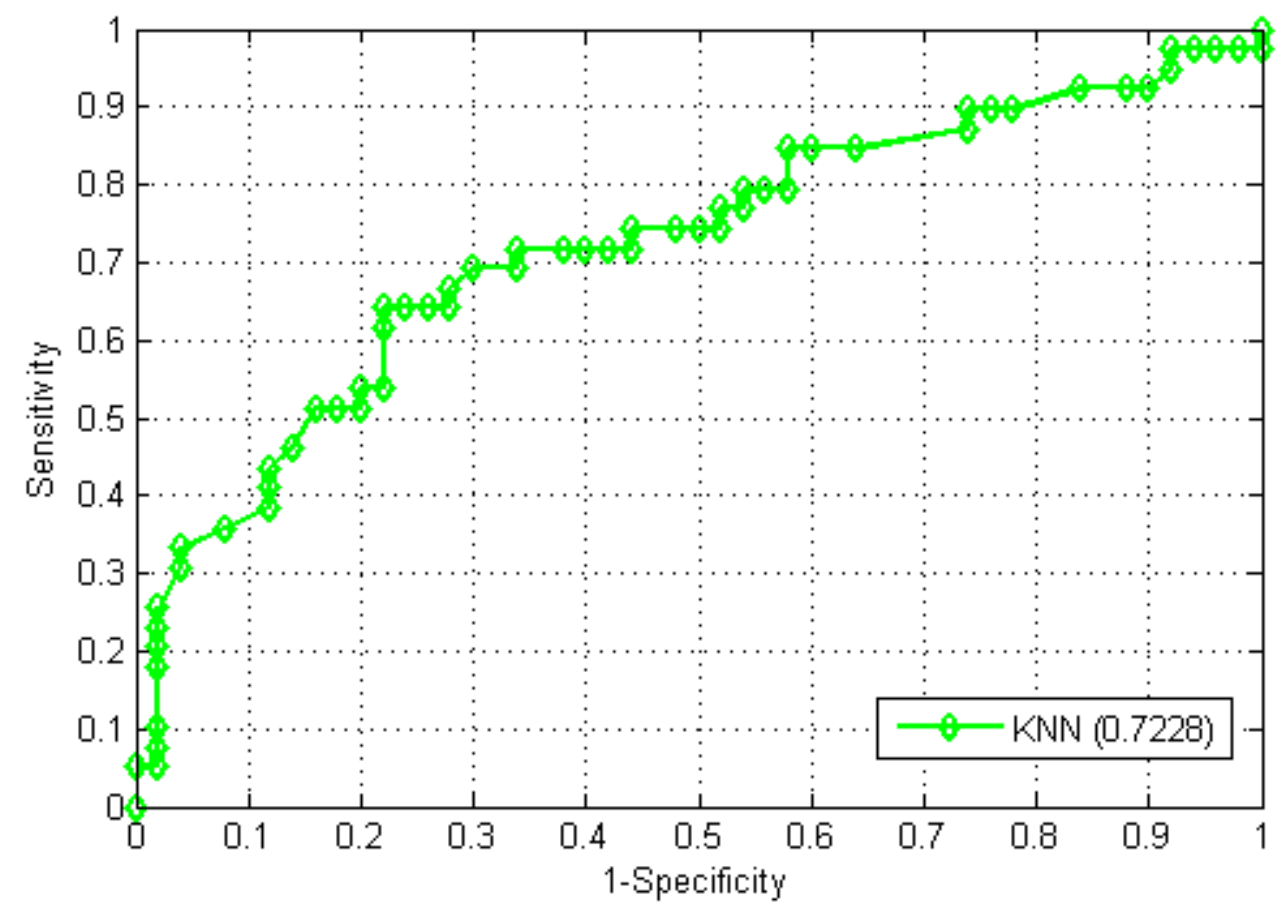

Figure 13: ROC curve of DIARETDB1 dataset.

method to handle illumination/shading variations caused by different fundus cameras.

\section{Acknowledgment}

This work were supported in part by grants from Oak Ridge National Laboratory (ORNL), and by the Regional Burgundy Council, France.

\section{References}

[1] M. Abràmoff, M. Garvin, M. Sonka, Retinal imaging and image analysis, Biomedical Engineering, IEEE Reviews in 3 (2010) 169-208.

[2] C. Baudoin, B. Lay, J. Klein, Automatic detection of microaneurysms in diabetic fluorescein angiography., Revue d'épidémiologie et de santé publique 32 (3-4) (1984) 254. 
[3] T. Spencer, J. A. Olson, K. C. McHardy, P. F. Sharp, J. V. Forrester, An image-processing strategy for the segmentation and quantification of microaneurysms in fluorescein angiograms of the ocular fundus, Comput. Biomed. Res. 29 (4) (1996) 284-302.

[4] A. J. Frame, P. E. Undrill, M. J. Cree, J. A. Olson, K. C. McHardy, P. F. Sharp, J. V. Forrester, A comparison of computer based classification methods applied to the detection of microaneurysms in ophthalmic fluorescein angiograms., Computers in biology and medicine 28 (3) (1998) $225-238$.

[5] M. Niemeijer, B. Van Ginneken, J. Staal, M. Suttorp-Schulten, M. Abràmoff, Automatic detection of red lesions in digital color fundus photographs, Medical Imaging, IEEE Transactions on 24 (5) (2005) 584-592.

[6] R. Gonzalez, E. Richard, Woods, digital image processing (2002).

[7] T. Spencer, R. Phillips, P. Sharp, J. Forrester, Automated detection and quantification of microaneurysms in fluorescein angiograms, Graefe's archive for clinical and experimental ophthalmology 230 (1) (1992) 3641.

[8] M. Cree, E. Gamble, D. Cornforth, Colour normalisation to reduce inter-patient and intra-patient variability in microaneurysm detection in colour retinal images.

[9] M. Foracchia, E. Grisan, A. Ruggeri, Luminosity and contrast normalization in retinal images, Medical Image Analysis 9 (3) (2005) 179-190.

[10] T. Walter, P. Massin, A. Erginay, R. Ordonez, C. Jeulin, J.-C. Klein, Automatic detection of microaneurysms in color fundus images, Medical Image Analysis 11 (6) (2007) 555 - 566.

[11] C. Köse, U. Şevik, C. İkibaş, H. Erdöl, Simple methods for segmentation and measurement of diabetic retinopathy lesions in retinal fundus images, Computer methods and programs in biomedicine 107 (2) (2012) $274-293$. 
[12] M. D. Saleh, C. Eswaran, An automated decision-support system for non-proliferative diabetic retinopathy disease based on mas and has detection, Computer methods and programs in biomedicine 108 (1) (2012) 186-196.

[13] C. Sánchez, R. Hornero, A. Mayo, M. García, M. Lopez, Mixture modelbased clustering and logistic regression for automatic detection of microaneurysms in retinal images, SPIE Medical Imaging 2009: ComputerAided Diagnosis 7260 (2009) 72601M.

[14] I. Lazar, A. Hajdu, R. Quareshi, Retinal microaneurysm detection based on intensity profile analysis, in: 8th International Conference on Applied Informatics, 2010.

[15] I. Lazar, A. Hajdu, Retinal microaneurysm detection through local rotating cross-section profile analysis, Medical Imaging, IEEE Transactions on 32 (2) (2013) 400-407.

[16] B. Zhang, X. Wu, J. You, Q. Li, F. Karray, Detection of microaneurysms using multi-scale correlation coefficients, Pattern Recognition 43 (6) (2010) 2237-2248.

[17] S. Abdelazeem, Micro-aneurysm detection using vessels removal and circular hough transform, in: Radio Science Conference, 2002. (NRSC 2002). Proceedings of the Nineteenth National, 2002, pp. $421-426$.

[18] A. Mizutani, C. Muramatsu, Y. Hatanaka, S. Suemori, T. Hara, H. Fujita, Automated microaneurysm detection method based on double ring filter in retinal fundus images, SPIE Medical Imaging 2009: ComputerAided Diagnosis 7260 (1) (2009) 72601N.

[19] D. Youssef, N. H. Solouma, Accurate detection of blood vessels improves the detection of exudates in color fundus images, Computer methods and programs in biomedicine.

[20] M. Fraz, S. Barman, P. Remagnino, A. Hoppe, A. Basit, B. Uyyanonvara, A. Rudnicka, C. Owen, An approach to localize the retinal blood vessels using bit planes and centerline detection, Computer Methods and Programs in Biomedicine 108 (2) (2012) 600 - 616. 
[21] M. M. Fraz, P. Remagnino, A. Hoppe, B. Uyyanonvara, A. R. Rudnicka, C. G. Owen, S. A. Barman, Blood vessel segmentation methodologies in retinal images-a survey, Computer methods and programs in biomedicine 108 (1) (2012) 407-433.

[22] G. Quellec, M. Lamard, P. Josselin, G. Cazuguel, B. Cochener, C. Roux, Optimal wavelet transform for the detection of microaneurysms in retina photographs, Medical Imaging, IEEE Transactions on 27 (9) (2008) $1230-1241$.

[23] L. Giancardo, F. Meriaudeau, T. Karnowski, K. Tobin, Y. Lic, E. Chaum, Microaneurysms detection with the radon cliff operator in retinal fundus images., in: Proc. of SPIE Vol, Vol. 7623, 2010, pp. $76230 \mathrm{U}-1$.

[24] L. Giancardo, F. Meriaudeau, T. Karnowski, Y. Li, K. Tobin, E. Chaum, Microaneurysm detection with radon transform-based classification on retina images, in: Engineering in Medicine and Biology Society, EMBC, 2011 Annual International Conference of the IEEE, IEEE, 2011, pp. 5939-5942.

[25] E. Grisan, A. Ruggeri, A hierarchical bayesian classification for nonvascular lesions detection in fundus images, in: EMBEC05, 3rd European Medical and Biological Engineering Conference, 2005.

[26] K. Ram, G. Joshi, J. Sivaswamy, A successive clutter-rejection-based approach for early detection of diabetic retinopathy, Biomedical Engineering, IEEE Transactions on 58 (3) (2011) 664-673.

[27] B. Antal, A. Hajdu, An ensemble-based system for microaneurysm detection and diabetic retinopathy grading, Biomedical Engineering, IEEE Transactions on 59 (6) (2012) 1720-1726.

[28] B. Antal, I. Lazar, A. Hajdu, An optimal voting scheme for microaneurysm candidate extractors using simulated annealing, in: Signal Processing and Multimedia Applications (SIGMAP), Proceedings of the 2010 International Conference on, IEEE, 2010, pp. 80-87.

[29] M. Niemeijer, B. Van Ginneken, M. Cree, A. Mizutani, G. Quellec, C. Sanchez, B. Zhang, R. Hornero, M. Lamard, C. Muramatsu, et al., 
Retinopathy online challenge: Automatic detection of microaneurysms in digital color fundus photographs, Medical Imaging, IEEE Transactions on 29 (1) (2010) 185-195.

[30] K. M. Adal, S. Ali, D. Sidibé, T. Karnowski, E. Chaum, F. Meriaudeau, Automated detection of microaneurysms using robust blob descriptors., in: Proc. of SPIE Vol, Vol. 8670, 2013.

[31] T. Lindeberg, Feature detection with automatic scale selection, International journal of computer vision 30 (2) (1998) 79-116.

[32] T. Lindeberg, Scale-space theory in computer vision, Springer, 1993.

[33] H. Bay, A. Ess, T. Tuytelaars, L. Van Gool, Speeded-up robust features (surf), Computer Vision and Image Understanding 110 (3) (2008) 346359.

[34] A. Blum, T. Mitchell, Combining labeled and unlabeled data with cotraining, in: Proceedings of the eleventh annual conference on Computational learning theory, ACM, 1998, pp. 92-100.

[35] X. Zhu, Semi-supervised learning literature survey, Tech. Rep. 1530, Computer Sciences, University of Wisconsin-Madison (2005).

[36] C. Chang, C. Lin, LIBSVM: A library for support vector machines, ACM Transactions on Intelligent Systems and Technology 2 (2011) 27:1-27:27, software available at http://www.csie.ntu.edu.tw/ cjlin/libsvm.

[37] Niemeijer, M. Abramoff, Retinopathy online challenge. (Accessed on November 2, 2012).

URL http://roc.healthcare.uiowa.edu/results.php

[38] T. Kauppi, V. Kalesnykiene, J.-K. Kamarainen, L. Lensu, I. Sorri, A. Raninen, R. Voutilainen, H. Uusitalo, H. Kälviäinen, J. Pietilä, Diaretdb1 diabetic retinopathy database and evaluation protocol, Proc. Medical Image Understanding and Analysis (MIUA) (2007) 61-65. 